\title{
High resolution strain measurements in highly disordered materials
}

\author{
Mark Sutton" \\ Physics Department, McGill University, Montréal, Quebec, Canada H3A 2T8 \\ Julien R. M. Lhermitte $\odot$ \\ Center for Functional Nanomaterials, Brookhaven National Laboratory, Upton, New York 11973, USA \\ Françoise Ehrburger-Dolle $\odot$ \\ Univ. Grenoble Alpes, CNRS, LIPhy, F-38000 Grenoble, France \\ Frédéric Livet \\ SIMaP, Grenoble INP-CNRS-UJF, Boîte Postale 75, F-38402 Saint Martin d'Hères Cedex, France
}

(Received 28 April 2020; accepted 12 January 2021; published 8 February 2021)

\begin{abstract}
The ability to measure small deformations or strains is useful for understanding many aspects of materials. Here, an alternate analysis of speckle x-ray diffraction peaks is presented in which the systematic shifts of the speckles are analyzed allowing for strain (or flow) patterns to be inferred. This speckle tracking technique measures strain patterns with an accuracy similar to x-ray single crystal measurements but in amorphous or highly disordered materials.
\end{abstract}

DOI: 10.1103/PhysRevResearch.3.013119

\section{INTRODUCTION}

The measurement of small deformations in samples is a useful method to characterize the properties of materials, soft materials in particular. The properties of soft condensed matter systems are often controlled by small mesoscale structural changes and/or deformations that have energy scales comparable to thermal energies. These changes are the origin of many of their viscoelastic properties. Deformations are typically measured via strain, or the geometrical relative displacements of elements in a body. Small deformations are hard to measure. High resolution strain has been measured using $\mathrm{x}$ rays and neutron scattering [1-3] and visible light [4-6]. However, these measurements are mostly limited to crystalline materials and/or macroscopic sections of samples. There are few measurement techniques involving amorphous materials with $\mathrm{x}$ rays. Nielsen et al. [3] applied a three-dimensional absorption tomography technique. This technique provides a high resolution three-dimensional picture of the static strain of materials. However, this technique requires measuring many diffraction patterns, potentially restricting its use for in situ measurements. In this paper an extension of x-ray photon correlation spectroscopy (XPCS) is proposed for in situ measurements of strain in amorphous

\footnotetext{
*Corresponding author: mark@physics.mcgill.ca

Published by the American Physical Society under the terms of the Creative Commons Attribution 4.0 International license. Further distribution of this work must maintain attribution to the author(s) and the published article's title, journal citation, and DOI.
}

materials. It measures a two-dimensional (2D) projection of the strain, but the technique requires taking only two coherent diffraction patterns. Since the analysis is somewhat involved, the emphasis of this paper is simply on the technique itself.

For a simple insight into the technique, imagine a deformation of the underlying space, $\vec{r}^{\prime}=M \cdot \vec{r}$. For small deformations, the transformation consists of a possible rigidbody displacement and a local distortion. From this it follows that the strain is the difference between $M$ and the identity matrix and related to the gradient of $M$ for small deformations. That is, $\Gamma_{s}=M-I$ is the strain tensor. When $M$ is independent of $\vec{r}$, a homogeneous deformation results. Since diffraction is measured in reciprocal space, we consider the effect on the density $\rho(\vec{r})$ and its Fourier transform $\rho(\vec{q})$. To get the Fourier transform of the deformed material $\rho^{\prime}(\vec{q})$,

$$
\rho^{\prime}(\vec{q})=\int \rho(M \cdot \vec{r}) e^{-i \vec{q} \cdot \vec{r}} d \vec{r}=\rho\left(M^{-T} \cdot \vec{q}\right) / \operatorname{det}(M)
$$

by simple substitution. This calculation assumes a negligible change in the volume of integration. Speckle sizes are determined by the diffraction volume, and this sets the scale determining when a deformation is small and for determining when the change in the volume of integration can be neglected. Another approach is given by solving the convection-advection diffusion equation by the method of characteristics and may be found in Fuller et al. [7]. So if we can relate features in $\rho(\vec{q})$ (or scattering intensity) before and after the deformation, their time-dependent shifts in reciprocal space are

$$
\Delta \vec{q}=M^{-T} \cdot \vec{q}-\vec{q}=-\Gamma_{s}^{T} \cdot \vec{q},
$$


where $M^{-T} \approx I-\Gamma_{s}^{T}$ for small deformations. For a simple velocity field

$$
\begin{aligned}
M(t) \cdot \vec{r} & =\vec{r}+\int_{0}^{t} \vec{v}(\vec{r}, \tau) d \tau \\
& =\vec{r}+\left(\vec{v}_{0}+\Gamma \cdot \vec{r}\right) t=\left(I+\Gamma_{s}\right) \cdot \vec{r},
\end{aligned}
$$

showing the strain tensor is related to the velocity gradient matrix $\Gamma=\nabla \vec{v}(\vec{r})$ for a velocity field. The rigid-body shift gives a phase factor $\exp \left(i \vec{v}_{0} \cdot \vec{q} \tau\right)$ due to the shift in $\vec{r}$ and will be ignored as it will not be seen in the scattered intensity. It is also assumed that the velocity field is in the steady state for the time of the measurement of each diffraction pattern.

Using these ideas for the analysis of diffraction patterns involves relating features in the starting diffraction pattern and the deformed diffraction pattern, measuring the shift in these wave vectors, and analyzing the shifts to obtain the strain matrix.

The illumination of a disordered material with a coherent source leads to a complicated interference pattern modulating the conventional (incoherent) diffraction pattern. This pattern is typically called a speckle pattern. The pattern reflects more than one length scale. There is a set of short length scales which reflect the scattering of the pieces or blocks which lead to longer distances in the diffraction pattern. These are also reflected in conventional x-ray diffraction, and their analysis is well developed. The speckles have short length scales in reciprocal space and arise due to the finite size of the beam. The rule of thumb is that the modulating speckle pattern has many peaks of widths $2 \pi / L$, where $L$ is the appropriate beam dimension on the sample. At each point in the diffraction pattern there is a contribution from each of the many blocks or subsections, each with its own phase factor randomly varying from 0 to $2 \pi$. These phases will add up to a well-defined phase under coherent illumination. If they add in phase, the result is constructive interference, and the intensity is high. Where they add up out of phase, intensity is low. Each speckle peak is somewhat like a local Bragg peak. Below we show that tracking the positions of speckles can be used to infer small deformations.

\section{ANALYSIS}

The shift in wave vectors after a deformation can be obtained from the following correlation function:

$$
g_{2}\left(\vec{q}_{0}, \Delta \vec{q}, \tau\right)=\frac{\langle I(\vec{q}, t) I(\vec{q}+\Delta \vec{q}, t+\tau)\rangle_{\vec{q}}}{\bar{I}^{2}} .
$$

Since each wave vector shifts by different amounts, the average over $\vec{q}$ is over a small region centered around $\vec{q}_{0}$. This assumes that the displacement is slowly varying in $\vec{q}$. Notice that when the shifts are time invariant or slow, it is possible to also average in time. When the scattering is sharply peaked, as for Bragg peaks and for speckles, one can measure $\Delta \vec{q}$ by following the local maxima. For Bragg peaks one measures the shifts of a few peaks. For speckles, the cross correlation measures the average shift of the speckles over the region averaged. As pointed out below, this is over many hundreds of speckles. The correlation function $g_{2}\left(\vec{q}_{0}, \Delta \vec{q}, \tau\right)$ is an extension of the intensity-intensity correlation function used in
XPCS. The time correlations for XPCS are calculated using $\Delta \vec{q}=0$.

The experiments were carried out at beamline 8-ID-I of the Advanced Photon Source. For this setup, the energy was $7.488 \mathrm{keV}(\lambda=1.663 \AA)$ monochromated by double-bounce $\mathrm{Ge}(111)$ crystals. The incident flux was $\approx 10^{9}$ photons $/ \mathrm{s}$ through a $20 \times 20 \mu \mathrm{m}^{2}$ aperture. A direct-illumination deepdepletion CCD (Princeton Instruments $1152 \times 1242$, 22.5- $\mu \mathrm{m}$ resolution) was used as an area detector. Each pixel in the area detector corresponds to $2.0 \times 10^{-5} \AA^{-1}$, which is close to the speckle size. The filled rubber samples were held in a vacuum chamber with an in situ tensile stress-strain cell. The exposure time per frame was $0.1 \mathrm{~s}$ recurring every $2.0 \mathrm{~s}$. Further details are given in Refs. [8,9].

The sample consists of a cross-linked elastomer (ethylene propylene diene monomer, rubber) filled with hydroxylated pyrogenic silica (AEROSIL 200 [10-12]). The volume fraction of silica is close to 0.16. In our measurements, the 1-mm-thick sheets are punched out to a classical dumbbell shape (width $=4 \mathrm{~mm}$ ). As described in Ref. [8], upon applying a step strain on the sample, the stress jumps and then slowly relaxes as the sample is held at constant strain. For this article, only one 400-s data set is presented for a sample which was stretched by $60 \%$, after which the macroscopic strain was fixed. The data collection for this run started approximately $1250 \mathrm{~s}$ after the application of the $60 \%$ strain step. These data are part of those presented in Ref. [8], where emphasis is on the understanding of the underlying science of elastomers. Here, since the analysis is somewhat involved, this article emphasizes the data analysis. Only a single run is described as this simplifies the discussion while still demonstrating the generality of the technique. The technique measures small strains in disordered materials and does not apply only to the elastomers used here. Using this analysis on the other data sets presented in Ref. [8] confirms the ideas presented here and should be used to study the viscoelastic properties of the elastomers which were studied.

The 200 frames of the data set have been sequentially averaged by 5 frames to produce 40 images which are analyzed below. To select the small regions of $\vec{q}$ for the cross correlations, the scattering images are decomposed into small wedges or bins of $|q|$ and $\phi$. The orientation of the detector is such that vertical on the detector is vertical on the sample. The azimuthal angle $\phi$ is $\operatorname{atan}\left(q_{\text {vert }} / q_{\mathrm{hor}}\right)$. The wedges are 20 pixels $\left(0.00040 \AA^{-1}\right)$ wide in $|\vec{q}|$ and $10^{\circ}$ in $\phi$. Cross correlations for all wedges with more than 1000 pixels up to a wave vector of $0.024 \AA^{-1}$ (1200 pixels) were calculated. This gave 376 nonoverlapping wedges. Bins are numbered with $\phi$ increasing for fixed $q$, and then $q$ increases for the next set of $\phi$. Figure 1 shows a typical bin (bin 57, $q=0.0090 \AA^{-1}, \phi=200^{\circ}$ ) and its cross correlation between the first averaged frames. As for each cross correlation [13,14], it has a peak amplitude of $1+\beta$ (speckle contrast) sitting on a background of height 1 . Each cross correlation is least-squares fit to a 2D Gaussian peak. Peak widths give the speckle size. The shift of the peak maximum gives the speckle movement. The cross correlation is calculated for integral pixel shifts, with no shift defining zero and points on each side being plus or minus a 1-pixel shift. If there is no peak shift, the cross correlation will be symmetric about zero. It is easy to see if a shift by a fraction 

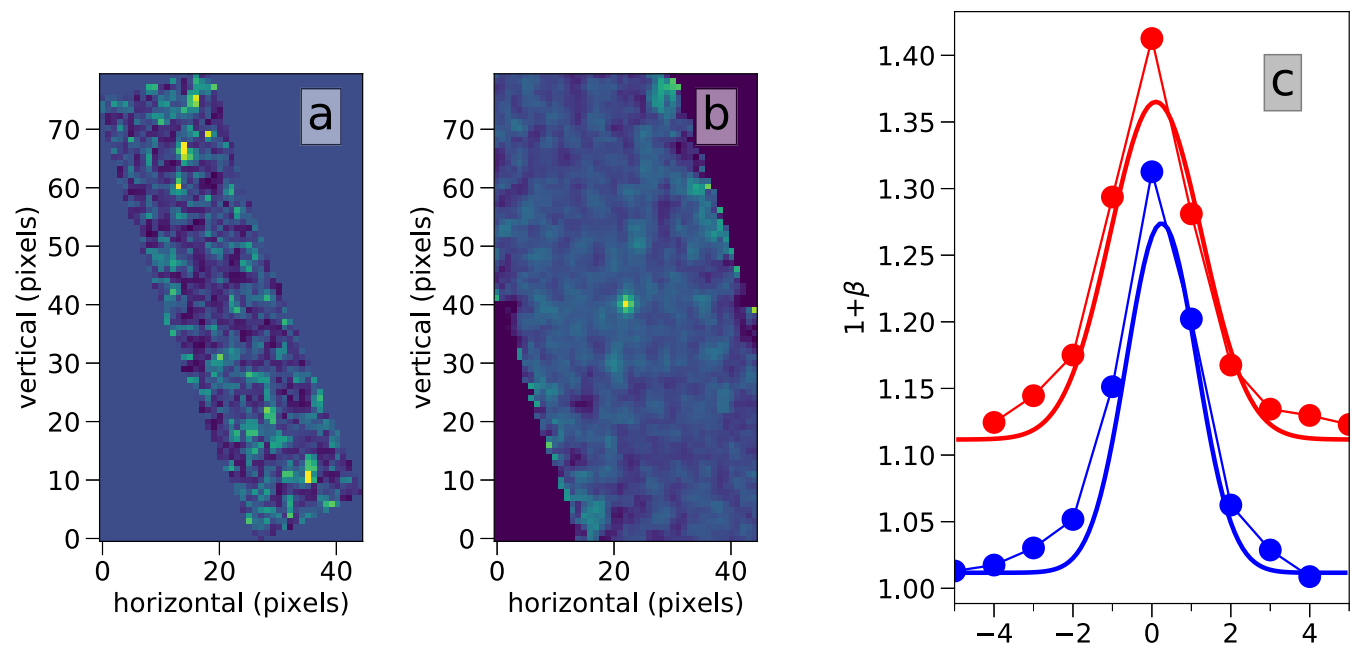

FIG. 1. (a) Speckle intensity for bin $57, q=0.009 \AA^{-1}, \phi=200^{\circ}$, as placed in a rectangular image. The underlying diffraction intensity is constant over the wedge, and the fractional intensity fluctuates by approximately $\pm \sqrt{0.3}=0.55$, reflecting the speckle variation. (b) Cross correlation between the first two averaged by five images. Scale can be determined from (c). (c) Line-outs around the central pixel of the cross correlation in the vertical (upper curves, offset by 0.1) and horizontal (lower curves) directions. The thick lines are for the 2D Gaussian that results from fitting the peak. The resulting shifts are 0.24 pixel in the horizontal and -0.10 pixel in the vertical and are reflected in the asymmetric placement of the measured points.

of a pixel exists as this leads to asymmetric values of the data at the \pm 1 positions, as shown in Fig. 1(c). Figure 1(c) also shows sections of the 2D Gaussian fit for this cross correlation. Note that for this example the center of the peak is offset by $(0.24,-0.10)$ pixels, or $\left(4.8 \times 10^{-6},-2.0 \times 10^{-6}\right) \AA^{-1}$, in $\left(q_{\text {hor }}, q_{\text {vert }}\right)$ from the center or the unshifted correlation point. The full widths at half maxima are 2.733 and 2.093 pixels in the horizontal and vertical directions, and $\beta=0.263$. A preliminary version of this analysis is given in Lhermitte's Ph.D. thesis [15].

Figure 2 shows the intensity of the time-averaged smallangle scattering (the blank rectangle in the top right is the beam stop). Superimposed is a representation of the speckle shifts between the first image and two other times. The shift for each bin is plotted as an arrow starting at the center of the

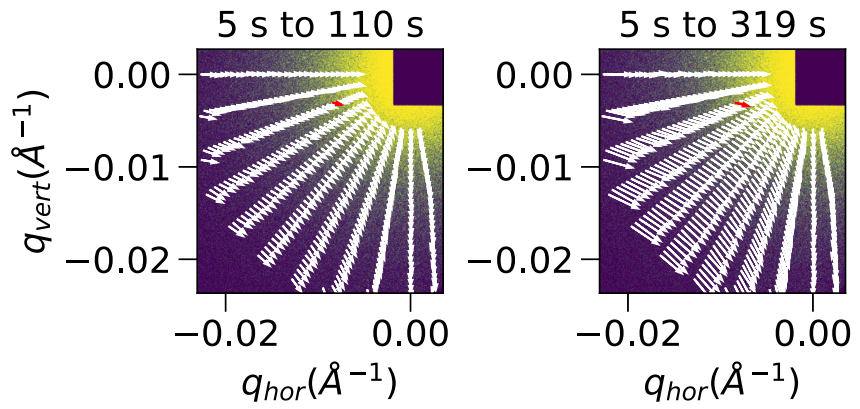

FIG. 2. Strain field for images from times 5 to $110 \mathrm{~s}$ and for images at 5 to $319 \mathrm{~s}$. Each local $q$ region has a shift in the speckle pattern given by the arrows, which are scaled up by 10 ( $q$ per pixel $=$ $\left.2.00 \times 10^{-5} \AA^{-1}\right)$. The coordinates of the shifts for the right panel are plotted in Fig. 5. The shifts are superimposed on the underlying small-angle $\mathrm{x}$-ray scattering intensity, and the blank rectangle in the top right corner is from the beam stop. For reference, bin 57's shift is shown with a different color. bin. Since the shifts are so small, the shift in pixels has been multiplied by 10 , where 1 pixel is $2.0 \times 10^{-5} \AA^{-1}$. One can immediately see that the relaxation of the filled rubber has a hyperbolic flow pattern. It is away from the beam center in the vertical $\left(\phi=270^{\circ}\right)$, which is along the tensile strain, and towards the beam center in the horizontal $\left(\phi=180^{\circ}\right)$. One also sees the shifts increase with $|\vec{q}|$. For reference, the shift for bin 57 is plotted with a different color.

Equation (4) is similar to the equation for XPCS except the analysis is only for the $\Delta \vec{q}=0$ term. XPCS has demonstrated its ability to measure time constants from microseconds to many hours. It has demonstrated that static disorder leads to speckle patterns unchanging in time, also for hours. Figure 3 shows $g_{2}(\tau)$ as one would calculate it in an XPCS

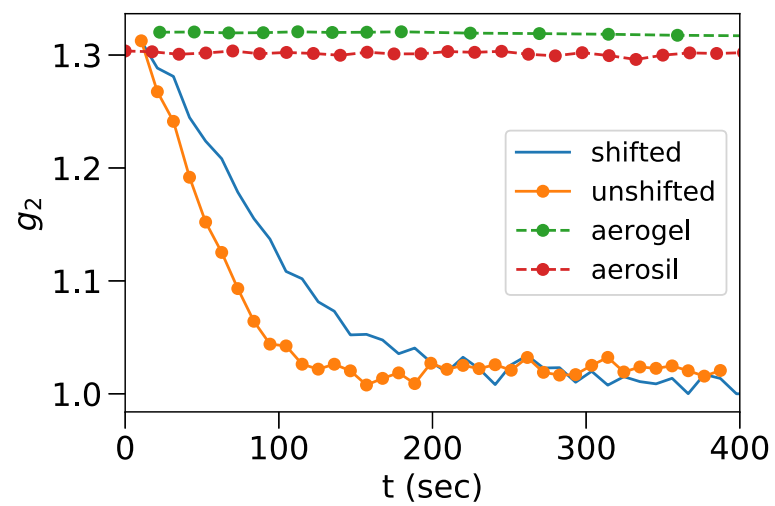

FIG. 3. A conventional calculation of $g_{2}$ is shown by the line with dots, labeled "unshifted" $(\Delta \vec{q}=0)$. A measurement following the in-plane shift observed on the detector is given by the solid line, labeled "shifted." For reference, it shows measured data from a static aerogel sample and for a packed AEROSIL 200 sample (from Ref. [8], Fig. 7(a)). The data analyzed are from the same $q-\phi$ bin as in Fig. 1. 


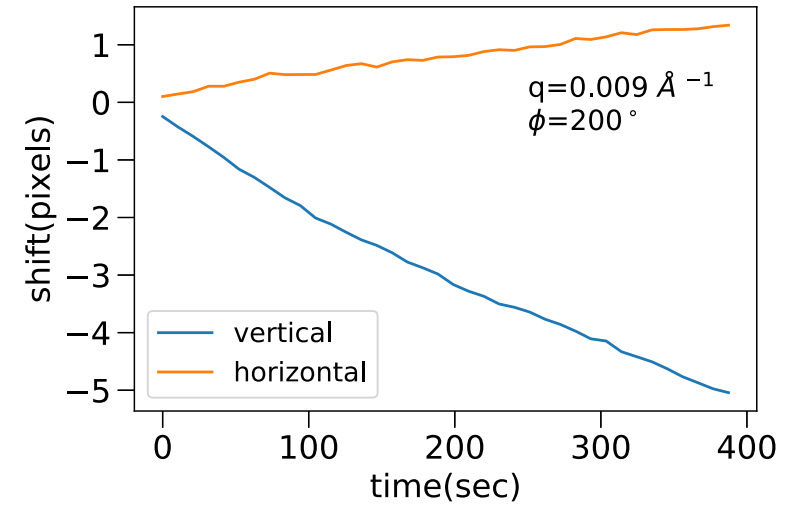

FIG. 4. The cumulative shift for bin 57 over the length of the time series. A pixel is $2.0 \times 10^{-5} \AA^{-1}$.

measurement. The slower decaying solid line in Fig. 3 is obtained by following the peak maximum from fitting the cross correlation of the first averaged by five patterns in time versus each consecutive averaged by five sequences of patterns [16]. The decay time of the slower curve is the average time it takes for speckles in bin 57 to move through the Ewald sphere in three-dimensional reciprocal space. To demonstrate stability, data points for $g_{2}$ from a silica aerogel and a packed AEROSIL 200 silica (Ref. [8], Fig. 7(a)) measured with the same setup are shown. They do not decay for the times shown. The cross-correlation analysis of the aerogel data shows the speckles do not shift in $\vec{q}$. This can also be inferred from Fig. 3 as any shift would cause $g_{2}$ to decay.

The fastest decaying curve in Fig. 3 is an example of the type of curves analyzed in Ref. [8] using conventional relaxation correlation functions. Figure 1(c) shows that speckles are approximately 2 pixels wide. Figure 4 shows that for about $100 \mathrm{~s}$ and longer the speckles for bin 57 have shifted by more than 2 pixels. This is the origin of the loss of correlation for time delays longer than $100 \mathrm{~s}$. This analysis shows that the decay in time for $g_{2}$ is due to the shift of the speckles and not to random diffusion. That the shifts increase linearly in $\vec{q}$ explains why the time constants vary as $1 / q$. It also shows that the time constants measured in Ref. [8] are indirect measures of velocity gradients. It is important to realize that the motion of the speckles is happening at constant macroscopic strain. The measured motion is accompanied by a changing macroscopic stress. The speckle motion is expected in all three dimensions. We expect the time for the speckle to move perpendicular to the detector plane to be comparable to the time of in-plane motion given the uniaxial nature of the applied strain. The small angle x-ray scattering (SAXS) with the speckle averaged away is equivalent to a conventional SAXS measurement. The SAXS pattern is isotropic and did not vary for the 200 images of this data set.

A cross correlation can be calculated for each bin and any pair of diffraction patterns. Only the shifts measured for each subsequent pair of patterns are used here. Single-frame cross correlations give quite acceptable correlations, but for this analysis five images are averaged and then correlated as this gives cleaner images. Since the correlations die away in minutes, the shifts are measured by cross correlating each nearest time pair of the averaged scattering. Using patterns

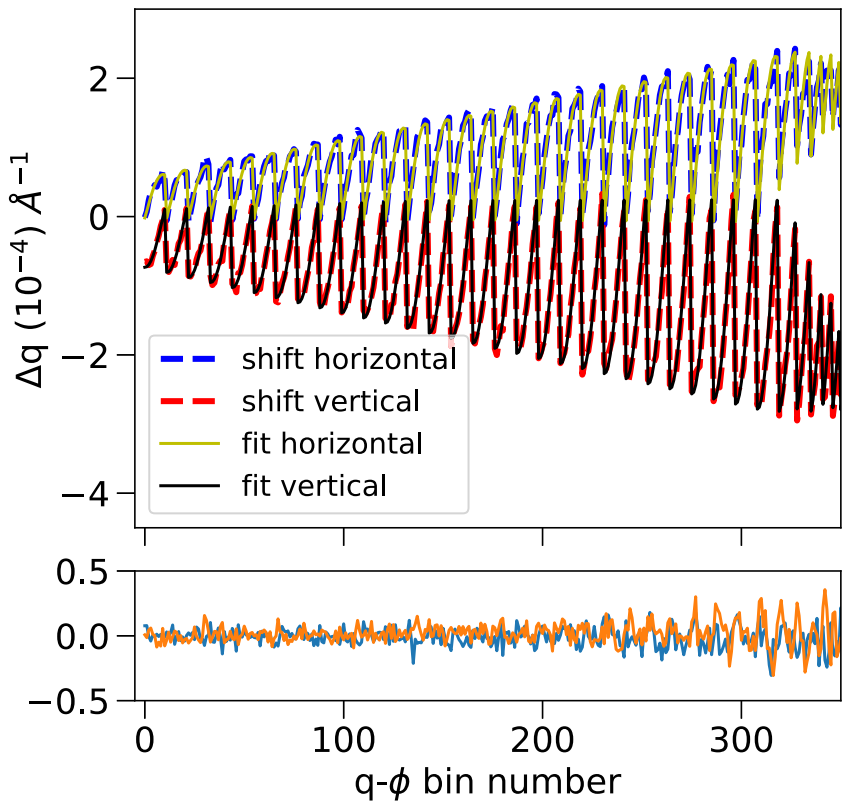

FIG. 5. Example of a two-parameter fit to a simple diagonal deformation pattern. The two components of the vector shift are plotted separately with dashed lines; positive curves are for the horizontal, and negative curves are for the vertical. The fitted values (solid lines) overlap with the data, and so the bottom panel shows the two sets of differences between the fits and the measurements. The vector field used in the fit is the same as in the right panel of Fig. 2 and corresponds to $\Gamma_{\text {vert }}=31.97 \times 10^{-6}$ and $\Gamma_{\text {hor }}=-26.75 \times 10^{-6}$ per second. These two parameters were used to generate the solid lines. The sawtooth nature of the data reflects the order of the bins in the diffraction pattern. For a given $q$ the bins increase in $\phi$, and then bins increase in $q$, giving the next tooth.

further separated in time leads to compatible shifts, but they have weaker correlations and are less accurate. Since the deformations are varying slowly across the whole run, it is better to measure nearest neighbor shifts and integrate the shifts for longer time intervals. Summing the shifts leads to a cumulative shift over the run and is shown in Fig. 4 for bin $57\left(q=0.009 \AA^{-1}, \phi=200^{\circ}\right)$.

For a given time delay and from the cumulative shifts in each bin, a vector field may be calculated. Two examples of these are plotted in Fig. 2. For each delay time slice, the vector field can be fit to a velocity gradient: $d \vec{q} / d t=-\Gamma \cdot \vec{q}$, where $d \vec{q}$ comes from the measured shifts and $d t$ is the time between images. Each vector field is well described by a diagonal 2D matrix. Since it is difficult to compare 2D vector field images, the quality of a typical fit is shown in Fig. 5 by a comparison between the fit and the measured vector components for the first 350 bins. This gives a quantitative representation of the quality of the fit. Since the fits agree well with the data, the white arrows on the right side of Fig. 2 also show this vector field in another representation. Explicitly, for this image the deformation is $\left(\Delta q_{\mathrm{hor}}, \Delta q_{\mathrm{vert}}\right)=\left(-0.0089 q_{\mathrm{hor}}, 0.0106 q_{\mathrm{vert}}\right)$, and the ratio $\Delta \vec{q} / \vec{q}$ gives the diagonal elements of the strain tensor. A simple two-parameter fit to all shifts of a given time delay fits all $q-\phi$ bins exceptionally well.

Figure 6 shows the evolution of the diagonal elements of the velocity gradient tensor $\Gamma$ as a function of time obtained 


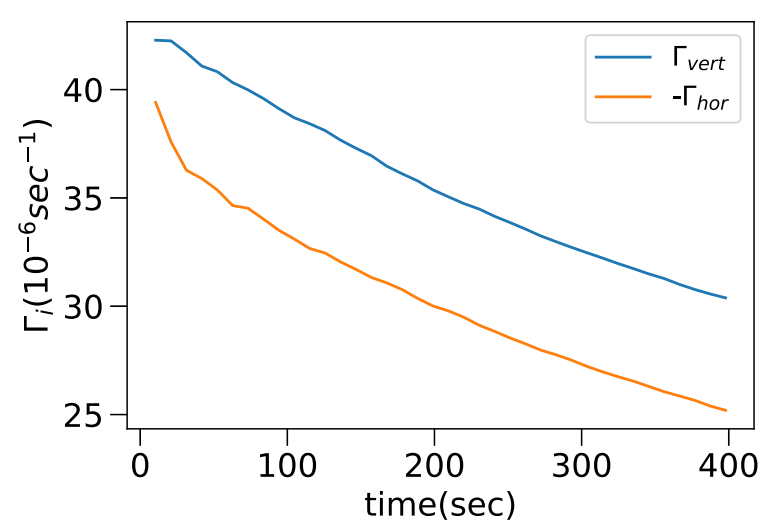

FIG. 6. Time evolution of the velocity gradient parameters obtained by fitting the vector fields over the run. The cumulative strain is the integral of $\Gamma$ over time.

from all vector field fits. Integrating this over time gives the strain as discussed above. Remember that here, $\Gamma$ is a velocity gradient tensor. For reference, $\Gamma_{\text {vert }}=35.0 \times 10^{-6} \mathrm{~s}^{-1}$ means that points $10 \mu \mathrm{m}$ apart in the vertical direction have a difference in vertical velocity of $3.5 \AA / \mathrm{s}$. These velocity gradients are quite compatible with the coarser "ballistic" velocity estimates using a compressed exponential correlation function based on Cipelletti et al. [17] (see Ehrburger-Dolle et al. for an earlier discussion of these data [8]). The evolution of $\Gamma$ with time reflects the slow viscoelastic relaxation in the silica-filled rubber while the sample is held at constant macroscopic strain.

\section{CONCLUSIONS}

In conclusion, it has been shown that a simple extension of XPCS can measure the projection of the strain tensor across the scattering volume. Since the dimensions of the beam are $20 \times 20 \mu \mathrm{m}^{2}$, this gives a submicron measurement of the local strain fields, and the strain precision is similar to what can be measured in a single-crystal measurement using the shifts in a Bragg peaks. It is stressed here that this analysis works for amorphous or highly disordered materials, in particular most heterogeneous soft matter systems. Details of the rheological implications for our samples and for the conventional XPCS analysis [18] from this approach are left for future work. It is worth pointing out that the decay of the XPCS signal in this system can be explained by the viscoelastic flow of the filled rubber, without any random Brownian motion.

We emphasize that the main result of this paper is that small strain fields in amorphous materials can be measured by cross correlating speckle patterns from before and after a change in strain. Nothing in the above analysis is specific to elastomers except maybe for the uniaxial approximation used to fit the vector field. Other distortions may require a different model. We emphasize that the strain measurement requires only two images with sufficient intensity to measure their speckle patterns and that the two images are separated in time by less than the time it takes for the speckles to move off the Ewald sphere [19]. Too large a change in strain during the exposure time of the images will smear the speckles and reduce sensitivity. Also, if the out-of-plane strain between the two images is too large, there will be no correlation, and the strain cannot be measured. For SAXS, movement along the beam in either the forward or backward direction has no in-plane component and so does not contribute to the diffraction pattern, but it can contribute to the motion of the speckle peaks. Also in-plane motions are averaged through the sample along the beam.

The advent of the new lattice structures that are being used to upgrade $\mathrm{x}$-ray synchrotrons will lead to an increase in coherence by two to three orders of magnitude. Also a new generation of x-ray area detectors with multikilohertz rates is becoming commercially available, and still faster ones are in development. The upgraded sources and the new detectors will enable speckle measurements to be easily performed with times on the order of microseconds. For the set of experiments upon which this analysis is reported these advances allow for measuring strains during the initial stage of deformation when the macroscopic strain is applied. We also point out that one of the nice features of this technique is one can easily measure nanometer-sized distortions and even slow measurements of these are extremely important for creep and aging studies in materials.

\section{ACKNOWLEDGMENT}

This research used resources of the Advanced Photon Source, a U.S. Department of Energy (DOE) Office of Science User Facility operated for the DOE Office of Science by Argonne National Laboratory under Contract No. DE-AC0206CH11357. We thank the staff of beamline 8-ID-I for their excellent help.
[1] I. Robinson and R. Harder, Coherent X-ray diffraction imaging of strain at the nanoscale, Nat. Mater. 8, 291 (2009).

[2] A. J. Allen, M. T. Hutchings, C. G. Windsor, and C. Andreani, Neutron diffraction methods for the study of residual stress fields, Adv. Phys. 34, 445 (1985).

[3] S. F. Nielsen, H. F. Poulsen, F. Beckmann, C. Thorning, and J. A. Wert, Measurements of plastic displacement gradient components in three dimensions using marker particles and synchrotron X-ray absorption microtomography, Acta Mater. 51, 2407 (2003).
[4] T. C. Chu, W. F. Ranson, and M. A. Sutton, Applications of digital-image-correlation techniques to experimental mechanics, Exp. Mech. 25, 232 (1985).

[5] M. A. Sutton, W. J. Wolters, W. H. Peters, W. F. Ranson, and S. R. McNeill, Determination of displacements using an improved digital correlation method, Image Vision Comput. 1, 133 (1983).

[6] I. Yamaguchi, A laser-speckle strain gauge, J. Phys. E 14, 1270 (1981).

[7] G. G. Fuller, J. M. Rallison, R. L. Schmidt, and L. G. Leal. The measurement of velocity gradients in laminar flow by 
homodyne light-scattering spectroscopy, J. Fluid Mech. 100, 555 (1980).

[8] F. Ehrburger-Dolle, I. Morfin, F. Bley, F. Livet, G. Heinrich, S. Richter, L. Piché, and M. Sutton, XPCS investigation of the dynamics of filler particles in stretched filled elastomers, Macromolecules 45, 8691 (2012).

[9] F. Ehrburger-Dolle, I. Morfin, F. Bley, F. Livet, G. Heinrich, L. Piché, and M. Sutton, Experimental clues of soft glassy rheology in strained filled elastomers, J. Polym. Sci. Part B 52, 647 (2014).

[10] T. P. Rieker, M. Hindermann-Bischoff, and F. Ehrburger-Dolle, Small-angle X-ray scattering study of the morphology of carbon black mass fractal aggregates in polymeric composites, Langmuir 16, 5588 (2000).

[11] F. Ehrburger-Dolle, M. Hindermann-Bischoff, E. Geissler, C. Rochas, F. Bley, and F. Livet, Role of fractal features in the structure-property relationships of carbon black filled polymers, MRS Proc. 661, KK7.4 (2001).

[12] F. Ehrburger-Dolle, F. Bley, E. Geissler, F. Livet, I. Morfin, and C. Rochas, Filler networks in elastomers, Macromol. Symp. 200, 157 (2003).

[13] Cross correlations were calculated by taking each wedge and placing it in a rectangular matrix with a size twice the tightest rectangle covering the wedge. Correlations were calculated using Fourier transforms by further extending the matrix to one with sizes that are multiples of 2, 3, and 5. This ensures the fast Fourier transform can be used. This rectangle has many zeros, including those outside the wedges and those due to bad or masked pixels. To take these into account, let $B$ be a rectangular matrix of zeros and ones identifying which pixels have a signal, and let $I$ be the matrix of the same size with the signal to be correlated. Then $g_{2}=\left[\left(I_{0} \otimes I_{1}\right) \times(B \otimes B)\right] /\left[\left(B \otimes I_{0}\right) \times\right.$ $\left(I_{1} \otimes B\right)$ ], where $\times$ is element by element multiplication [14]. The denominator amounts to symmetric normalization.

[14] D. Padfield, Masked object registration in the Fourier domain, IEEE Trans. Image Process. 21, 2706 (2012).

[15] J. Lhermitte, Using coherent small angle xray scattering to measure velocity fields and random motion, Ph.D. thesis, McGill University, 2011.

[16] The speckle shifted contrast is the amplitude of the 2D Gaussian fit to the cross correlation of the two images for the given time delay.

[17] L. Cipelletti, L. Ramos, S. Manley, E. Pitard, D. A. Weitz, E. E. Pashkovski, and M. Johansson, Universal non-diffusive slow dynamics in aging soft matter, Faraday Discuss. 123, 237 (2003).

[18] F. Ehrburger-Dolle, I. Morfin, F. Bley, F. Livet, G. Heinrich, Y. Chushkin, and M. Sutton, Anisotropic and heterogeneous dynamics in stretched elastomer nanocomposites, Soft Matter 15, 3796 (2019).

[19] As long as there is partial contrast, there will be speckles in the diffraction pattern and thus peaks. Being able to follow the shift of peaks in the diffraction pattern is all that is required for the analysis. The strain sensitivity will obviously be reduced for highly undersampled speckles as a larger shift in reciprocal space will be required to move the speckle with respect to the pixel size. It will also be more likely that a shift off the Ewald sphere will cause a loss in correlation. It thus helps if the intrinsic speckle size, which is determined by the diffraction volume, is comparable to the pixel size on the area detector. Speckles do not need to be oversampled. 